\title{
COMPARISON OF THE EFFECTS OF TWO DIFFERENT ANTIOXIDANT COMBINATIONS ON SPERM PARAMETERS
}

\author{
Mehmet Yariş ${ }^{1}$, Nebil Akdogan², Murat Ozturk ${ }^{3}$, and Aliseydi Bozkurt ${ }^{4}$ \\ ${ }^{1}$ ozel genesis hospital \\ ${ }^{2}$ Cukurova University Faculty of Medicine \\ ${ }^{3}$ Bursa Yuksek Ihtisas Training and Research Hospital \\ ${ }^{4}$ Erzincan University, Mengucek Gazi Research and Training Hospital
}

February 19, 2021

\begin{abstract}
Male infertility may be the result of some identifiable conditions (varicocele, cryptorchidism, hypogonadism, and genetic abnormalities), but there is no identified cause in 30-40\% of infertile men. Studies show that the use of antioxidants has a beneficial effect on fertility, especially on sperm quality, and therefore recommended as a potentially effective treatment for male infertility. However, it is argued that this treatment is not based on sufficient evidence and has no effect on the rate of healthy pregnancy. Aim: In this study, two different antioxidant combinations with different doses and contents were evaluated in terms of their effect on sperm parameters. Materials and Methods: A total of 122 patients diagnosed with idiopathic infertility were enrolled in our multicenter study. The patients were divided into two different groups: The first group used Proxeed Plus sachets $2 \times 1$ and the second group used Carniact Duo tablets $2 \mathrm{x} 1$ for six months. The total semen volume, total sperm count and concentration, motile sperm count, and morphological findings of the patients were compared at the end of the six months. The mean age of the patients participating in the study was $30.8 \pm 6.05$ years. No significant difference was found between the two groups in terms of baseline sperm count. Results: There was a significant difference between the baseline and sixth-month values of the patients using both combinations. However, no significant statistical difference was found between the groups according to the sixth-month data. Conclusion: The combinations of both antioxidants had a positive effect on sperm parameters, and the use of different doses and contents had a similar effect. Keywords: Antioxidants, Infertility, Vitamins, Spermiogram,
\end{abstract}

Original Article

COMPARISON OF THE EFFECTS OF TWO DIFFERENT ANTIOXIDANT COMBINATIONS ON SPERM PARAMETERS

Mehmet Yaris ${ }^{1}$, Nebil Akdogan² ${ }^{2}$ Murat Öztürk ${ }^{3}$, Aliseydi Bozkurt ${ }^{4}$,

1. Genesis Private Hospital, Department of Urology, Diyarbakir, Turkey

2. Cukurova Universty Faculty of Medicine, Department of Urology, Adana, Turkey

3. Bursa High Specialization Research and Training Hospital, Department of Urology, Bursa, Turkey

4. Erzincan University, Mengucek Gazi Research and Training Hospital, Department of Urology, Erzincan, Turkey

\section{Corresponding Author:}

Mehmet YARIS, M.D., Genesis Private Hospital, Department of Urology,

Phone:+9005057760736 Email: drmyaris@gmail.com

Adress: Kayapinar/Diyarbakir / TURKEY 
Disclosures: The authors declare that they have no relevant or material financial interests that relate to the research described in this paper.

Running Head: "antioxidant combinations"

Key Words: Antioxidants, Infertility, Vitamins, Spermiogram

\section{Abstract}

Male infertility may be the result of some identifiable conditions (varicocele, undescended testicle, hypogonadism, and genetic abnormalities), but there is no identified cause in $30-40 \%$ of infertile men. Studies show that the use of antioxidants has a beneficial effect on fertility, especially on sperm quality, and therefore recommended as a potentially effective treatment for male infertility. However, it is argued that this treatment is not based on sufficient evidence and has no effect on the rate of healthy pregnancy.

Aim: In this study, two different antioxidant combinations with different doses and contents were evaluated in terms of their effect on sperm parameters.

Materials and Methods: A total of 122 patients diagnosed with idiopathic infertility were enrolled in our multicenter study. The patients were divided into two different groups: The first group used Proxeed Plus sachets $2 \mathrm{x} 1$ (L-carnitine $1 \mathrm{~g}$, acetyl-L-carnitine $0.5 \mathrm{~g}$, fructose $1 \mathrm{~g}$, citric acid $0.50 \mathrm{mg}$, selenium $50 \mathrm{mcg}$, coenzyme Q10 $20 \mathrm{mg}$, vitamin C $90 \mathrm{mg}$, zinc $10 \mathrm{mg}$, folic acid $200 \mathrm{mcg}$, and vitamin B12 $1.5 \mathrm{mcg}$ ) and the second group used Carniact Duo tablets 2x1 ((L-carnitine $500 \mathrm{mg}$, selenium $50 \mathrm{mcg}$, coenzyme Q10 $20 \mathrm{mg}$, vitamin C $60 \mathrm{mg}$, zinc $15 \mathrm{mg}$, folic acid $400 \mathrm{mcg}$, vitamin $\mathrm{E}$ and ginseng $15 \mathrm{mcg}$ ) for six months. The total semen volume, total sperm count and concentration, motile sperm count, and morphological findings of the patients were compared at the end of the six months. The mean age of the patients participating in the study was $30.8 \pm 6.05$ years.

Results: No significant difference was found between the two groups in terms of baseline sperm count. There was a significant difference between the baseline and sixth-month values of the patients using both combinations. However, no significant statistical difference was found between the groups according to the sixth-month data.

Conclusion: The combinations of both antioxidants had a positive effect on sperm parameters, and the use of different doses and contents had a similar effect.

Keywords: Antioxidants, Infertility, Vitamins, Spermiogram,

\section{What is known?}

The studies analyzing the impact of antioxidants on sperm parameters have reached so far contradictory results. In particularly, there is no common standard and explicit suggestion for the doses and compounds of antioxidants for the treatment of male infertility.

\section{What is new?}

In this study, we attempted to determine the effect of antioxidant (food) supplements on semen quality and to compare two different molecules. We compared for the first time two different combination and doses of antioxidants. These different content and doses of antioxidant produced the same impact on sperm parameters.

\section{Introduction}

Infertility is defined as the inability of couples to have children despite one year of unprotected sexual intercourse (Sharlip et al. , 2002). The worldwide incidence of infertility is approximately 15\%, and male infertility accounts for $50 \%$ of all cases (WHO, 2000). Male infertility can be linked to a variety of conditions including congenital factors, varicocele, undescended testicle, testicular cancer, hypogonadism, infection, autoimmune diseases, systemic diseases, and genetic abnormalities. In about 30 to $40 \%$ of cases, there is no identified cause, which is referred to as idiopathic oligoasthenoteratozoospermia (OAT) (Nieschlag, Behre, 
\& Nieschlag, 2010). In men without any disease that may affect fertility, idiopathic OAT is diagnosed based on the combination of a low sperm concentration $(<15 \times 106 / \mathrm{mL})$, reduced motility (progressive motility $<32 \%$ and total motility $<40 \%)$ and abnormally shaped spermatozoa $(<30 \%$ normal morphology according to the 2010 World Health Organization criteria and $<4 \%$ normal morphology according to the Kruger strict criteria) (Cooper et al., 2010). Levels of reactive oxygen species (ROS) have also been associated with male infertility, and studies have reported higher levels of ROS and suppressed antioxidant capacity in the semen of infertile men compared to their fertile counterparts (Dorostghoal et al.2017, Agarwal et al., 2014). ROS are normally found in seminal plasma to some extent since they are necessary for capacitation, acrosome reaction, and fertilization, but spermatozoa are also vulnerable to oxygen radicals because plasma membranes and cytoplasm are rich in polyunsaturated fatty acids (Agarwal et al., 2014). In particular, high ROS exposure leads to functional changes that result in membrane damage, membrane instability, and cell death (6-Agarwal et al., 2014). Oxidative stress (OS) occurs when in the presence of an imbalance between oxidants and antioxidants (Agarwal, Hamada and Esteves, 2012).In general, an oxidative environment can lead to cellular degeneration with apoptosis or necrosis, and a reduced environment can support cell survival (Durackova et al., 2014). Therefore, a therapeutic strategy is to use supplements in order to enhance sperm energy metabolism, minimize sperm-free radical damage, and improve cellular processes associated with sperm formation and maturation. Many studies have confirmed the beneficial effect of antioxidants on at least one of the semen parameters, but the greatest effect has been determined in relation to sperm motility. In most of these studies, multiple combinations of antioxidants were used, but the optimal dose of one or more antioxidants was not defined. ( Imamovic Kumalic S, Pinter B.,2014 ).Several clinical studies have shown that the oral intake of L-carnitine and acetyl-l-carnitine in asthenozoospermic patients increases the percentage of motile spermatozoa, fast forward motility, mean velocity, sperm motility, and linearity (Balercia et al., 2005; Lenzi et al., 2004). It has been reported that the use of selenium, coenzyme Q10 (CoQ10), citric acid, vitamin C, vitamin B12, and zinc has positive effects on spermatozoa (Busetto et al., 2018).

Although many studies have noted the positive effects of the use of antioxidants, it has not been determined exactly which combination should be used and at which ratio. In this study, we investigated the effects of two different antioxidant combinations on sperm parameters in patients with idiopathic OAT and whether the use of each combination resulted in different effects.

\section{Materials and Methods}

The data from 122 patients were included in this multi-center (4 insutitions) prospective study, which was conducted between December 2018 and September 2020 to determine the effect of antioxidant (food) supplements on semen quality and to compare two different molecules. Infertile patients diagnosed with idiopathic infertility presenting with oligo and/or asthenia and/or teratospermia were included. The inclusion criteria were determined as the use of supplements for six months and the absence of any disease to explain the etiology of infertility. The consent for the study was obtained from the local ethics committee of Erzincan University (approval number: 000009/02).

The patients were divided into two groups. The formula of Proxeed Plus (Sigma-Tau HealthScience, Utrecht, the Netherlands) used in the first group contained 1,000 mg l-carnitine, $725 \mathrm{mg}$ fumarate, $500 \mathrm{mg}$ acetyll-carnitine, 1,000 mg fructose, $20 \mathrm{mg}$ CoQ10, $90 \mathrm{mg}$ vitamin C, $10 \mathrm{mg}$ zinc, $200 \mu \mathrm{g}$ folic acid, and $1.5 \mu \mathrm{g}$ vitamin B12. The second group used Carniact Duo tb (MG Farma Health Science, Mersin, Turkey), which contained L-carnitine $500 \mathrm{mg}$, selenium $50 \mathrm{mcg}$, coenzyme Q10 $20 \mathrm{mg}$, vitamin C $60 \mathrm{mg}$, zinc $15 \mathrm{mg}$, folic acid $400 \mathrm{mcg}$, vitamin E $30 \mathrm{mg}$ and ginseng $15 \mathrm{mcg}$. A spermiogram evaluation was performed at the beginning of the treatment to evaluate the semen parameters of each patient. At the end of six months of treatment, consecutive semen samples were collected. The pre-treatment semen samples, demographic data, physical examination blood pressure, anamnesis and previous/concurrent treatments were recorded (Table 1). Semen samples were collected after three-five days of sexual abstinence. Ejaculate volume, total sperm count, and total and progressive motility, as well as normal sperm morphology were evaluated according to the WHO guidelines (2010; 5th edition guidelines).

Table 1 : Demographic characteristics and comparisons of groups 


\begin{tabular}{llllllll}
\hline & Carniact & Carniact & Proxeed & Proxeed & Total & Total & $\mathrm{p}$ \\
& $\mathrm{n}$ & $\%$ & $\mathrm{n}$ & $\%$ & $\mathrm{n}$ & $\%$ & \\
Diabetes Mellitus & 0 & $(, 00)$ & 0 & $(, 00)$ & 0 & $(, 00)$ & $* * * 1$ \\
Hypertansion & 0 & $(, 00)$ & 6 & $(10,00)$ & 6 & $(4,92)$ & $\mathbf{0 , 0 1 1}^{\mathbf{1}}$ \\
Cigarette & 24 & $(38,71)$ & 22 & $(36,67)$ & 46 & $(37,70)$ & $0,816^{1}$ \\
Alchole & 9 & $(14,52)$ & 9 & $(15,00)$ & 18 & $(14,75)$ & $0,940^{1}$ \\
Pragnancy & 4 & $(6,45)$ & 3 & $(5,00)$ & 7 & $(5,74)$ & $0,730^{1}$ \\
Age & $31,18 \pm 6,22$ & 29,50 & $30,42 \pm 5,89$ & 29,50 & $30,80 \pm 6,05$ & 29,50 & $0,474^{2}$ \\
Testosterone & $5,75 \pm 2,56$ & 5,35 & $6,02 \pm 4,23$ & 4,00 & $5,89 \pm 3,52$ & 4,48 & $0,159^{2}$ \\
FSH & $6,17 \pm 4,19$ & 4,78 & $6,13 \pm 1,89$ & 6,00 & $5,89 \pm 3,52$ & 5,50 & $0,169^{2}$ \\
Prolactine & $10,73 \pm 3,01$ & 11,00 & $12,50 \pm 6,49$ & 10,00 & $5,89 \pm 3,52$ & 10,90 & $0,824^{2}$ \\
LH & $5,52 \pm 3,17$ & 4,80 & $5,12 \pm 3,30$ & 3,66 & $5,31 \pm 3,22$ & 3,85 & $0,451^{2}$ \\
Estradiol & $22,66 \pm 15,01$ & 22,40 & $20,55 \pm 7,83$ & 20,00 & $21,78 \pm 12,46$ & 20,50 & $0,892^{2}$ \\
\hline
\end{tabular}

FSH: follicle-stimulating hormone; LH: luteinizing hormone

The patients included in our study were men aged 18-50 years with a history of infertility for more than one year, who presented with oligo and/or asthenia and/or teratospermia, but no varicocele or other disease that could affect fertility. A complete examination was performed in each patient to exclude other causes of infertility (anamnesis, physical examination, scrotal Doppler ultrasound, and hormone analysis). The fertile female partners were under the age of 40 years and had regular menstrual cycles, and the couples were not intending to undergo a fertility-related procedure for the following 90 days.

The exclusion criteria were known hypersensitivity to any treatment compound, presence of undescended testicular or history of testicular cancer, hormonal diseases, history of post-pubertal mumps, genitourinary surgery, obstructive azoospermia or obstructive pathologies of the urogenital system, autoimmune diseases, cystic fibrosis, any treatment that would affect fertility within the last six months, excessive alcohol consumption or regular use of illegal or recreational drugs, following a special diet, participation in any clinical research, and any other condition that could put the patient at risk by participating in the study according to the researcher, and patients participating in any other clinical trial.

The endpoints of the study were sperm concentration, semen volume, total sperm count, total motility, progressive motility, and percentage of normal sperm morphology.

Statistical analysis:

Statistical analyses were carried out using SPSS version 17 . The normal distribution of variables was examined using histogram graphics and the Kolmogorov-Smirnov test. Mean, standard deviation and median values were used when presenting descriptive statistics.

For the inter-group comparison of data that did not show a normal distribution (non-parametric), the Mann-Whitney U test was conducted. Changes in measured values within the groups were evaluated with the Wilcoxon test. Results where the p value was below 0.05 were evaluated as statistically significant.

\section{Results:}

The mean age of the patients participating in the study was $30.8+-6.05$ years. The mean baseline total sperm count was 53.86 million in the Proxeed group and 49.82 million in the Carniact group, with no significant difference between the groups $(\mathrm{p}>0.05)$. However, there was a statistically significant difference between the two groups in terms of the pre-treatment baseline sperm motility and morphology parameters $(\mathrm{p}<0.05)$ (Table 2). There was a significant difference between the baseline and sixth-month total sperm counts of the patients in each of the two groups $(\mathrm{p}<0.05)$ (Table 3 and 4$)$. However, no significant changes were found in total semen volume in the Proxeed group and non-progressive motility in the Carniact group 
after treatment $(\mathrm{p}>0.05)$. There was also no statistically significant difference between the two groups in terms of post-treatment parameters $(\mathrm{p}>0.05)$ (Table 5).

Table 2 : Comparision groups before treatment

\begin{tabular}{llllllll}
\hline Baseline & Carniact & Carniact & Carniact & Proxeed & Proxeed & Proxeed & $\mathrm{p}$ \\
& Mean & SD & Median & Mean & SD & Median & \\
Total semen volume, ml & 2.67 & \pm 1.25 & 2.10 & 2.98 & \pm 1.14 & 3.00 & 0.058 \\
Total sperm count & 49.82 & \pm 40.29 & 38.00 & 53.86 & \pm 44.18 & 40.00 & 0.667 \\
Sperm concentration & 20.45 & \pm 20.17 & 13.00 & 20.94 & \pm 14.15 & 18.00 & 0.226 \\
Total motile sperm count & 38.06 & \pm 17.07 & 40.00 & 36.42 & \pm 15.54 & 38.00 & 0.149 \\
Fast forward motility (a) & 6.94 & \pm 9.07 & 2.50 & 13.23 & \pm 8.08 & 14.00 & 0.000 \\
Non-progressive motility (b) & 30.32 & \pm 15.55 & 30.00 & 23.35 & \pm 9.41 & 22.50 & 0.001 \\
Morphology & 1.55 & \pm 2.18 & .00 & 3.50 & \pm 3.16 & 2.00 & 0.000 \\
\hline
\end{tabular}

Mann-Whitney U test

Table 3 : Comparison of baseline and post-treatment values of the Carniact groups

\begin{tabular}{llllllll}
\hline Carniact Group & Baseline & Baseline & Baseline & Month 6 & Month 6 & Month 6 & p \\
& Mean & SD & Median & Mean & SD & Median & \\
Total semen volume, ml & 2.67 & \pm 1.25 & 2.10 & 3.13 & \pm 1.44 & 3.00 & 0.000 \\
Total sperm count & 49.82 & \pm 40.29 & 38.00 & 100.94 & \pm 68.75 & 90.00 & 0.000 \\
Sperm concentration & 20.45 & \pm 20.17 & 13.00 & 35.67 & \pm 25.93 & 28.00 & 0.000 \\
Total motile sperm count & 38.06 & \pm 17.07 & 40.00 & 49.44 & \pm 16.92 & 50.00 & 0.000 \\
Fast forward motility (a) & 6.94 & \pm 9.07 & 2.50 & 21.48 & \pm 17.90 & 20.00 & 0.000 \\
Non-progressive motility (b) & 30.32 & \pm 15.55 & 30.00 & 29.44 & \pm 15.10 & 30.00 & 0.703 \\
Morphology & 1.55 & \pm 2.18 & .00 & 5.61 & \pm 2.04 & 6.00 & 0.000 \\
\hline
\end{tabular}

Wilcoxon test

Table 4 : Comparison of baseline and post-treatment values of the Proxeed group

\begin{tabular}{llllllll}
\hline Proxeed Group & Baseline & Baseline & Baseline & Month 6 & Month 6 & Month 6 & $\mathrm{p}$ \\
& Mean & SD & Median & Mean & SD & Median & \\
Total semen volume, ml & 2.98 & \pm 1.14 & 3.00 & 3.13 & \pm 1.03 & 3.00 & 0.073 \\
Total sperm count & 53.86 & \pm 44.18 & 40.00 & 99.58 & \pm 69.22 & 80.00 & 0.000 \\
Sperm concentration & 20.94 & \pm 14.15 & 18.00 & 32.31 & \pm 19.97 & 29.00 & 0.000 \\
Total motile sperm count & 36.42 & \pm 15.54 & 38.00 & 50.50 & \pm 16.36 & 50.00 & 0.000 \\
Fast forward motility (a) & 13.23 & \pm 8.08 & 14.00 & 22.70 & \pm 17.55 & 20.00 & 0.000 \\
Non-progressive motility (b) & 23.35 & \pm 9.41 & 22.50 & 27.98 & \pm 16.15 & 29.00 & 0.029 \\
Morphology & 3.50 & \pm 3.16 & 2.00 & 5.71 & \pm 2.22 & 6.00 & 0.008 \\
\hline
\end{tabular}

Wilcoxon test

Table 5 : Comparision groups post treatment

\begin{tabular}{llllllll}
\hline Month 6 & Carniact & Carniact & Carniact & Proxeed & Proxeed & Proxeed & $\mathrm{p}$ \\
& Mean & SD & Median & Mean & SD & Median & \\
Total semen volume, ml & 3.13 & \pm 1.44 & 3.00 & 3.13 & \pm 1.03 & 3.00 & 0.363 \\
Total sperm count & 100.94 & \pm 68.75 & 90.00 & 99.58 & \pm 69.22 & 80.00 & 0.826
\end{tabular}




\begin{tabular}{llllllll} 
Sperm concentration & 35.67 & \pm 25.93 & 28.00 & 32.31 & \pm 19.97 & 29.00 & 0.856 \\
Total motile sperm count & 49.44 & \pm 16.92 & 50.00 & 50.50 & \pm 16.36 & 50.00 & 0.734 \\
Fast forward motility (a) & 21.48 & \pm 17.90 & 20.00 & 22.70 & \pm 17.55 & 20.00 & 0.637 \\
Non-progressive motility (b) & 29.44 & \pm 15.10 & 30.00 & 27.98 & \pm 16.15 & 29.00 & 0.064 \\
Morphology & 5.61 & \pm 2.04 & 6.00 & 5.71 & \pm 2.22 & 6.00 & 0.872 \\
\hline
\end{tabular}

Mann-Whitney U test

\section{Discussion}

In this study, we found that antioxidant treatments had a positive effect on sperm parameters and that the two different molecule combinations had similar effects. Male infertility may be the result of some identifiable conditions (varicocele, cryptorchidism, hypogonadism, and genetic abnormalities), but no cause can be identified in 30-40\% of infertile men. Such idiopathic infertility is characterized by decreased spermatozoa, decreased sperm motility, and abnormal sperm morphology in men that have no history of fertility-impairing disease and normal findings in physical examination and laboratory tests (Cooper et al., 2010).

It is assumed that various factors may be effective in idiopathic OAT, including endocrine disorders, genetic abnormalities, and ROS (Jungwirth et al., 2013). High ROS levels and oxidative stress have been associated with sperm DNA damage, decreased sperm motility, and impaired fertilization and embryo development (Agarwal et al., 2014). Since ROS damages lipids, amino acids, carbohydrates, proteins, and DNA of spermatozoa, this also affects sperm function (Agarwal et al., 2003). Therefore, targeting OS is considered to be a strategy to increase fertility and spermatozoa count and quality. Studies have shown that in addition to vitamins (mainly A, B, C and E) and non-enzymatic antioxidants including glutathione, metabolic coenzymes such as pantothenic acid, CoQ10 and carnitines (l-carnitine and acetyl-l-carnitine) and micronutrients (zinc, selenium, and copper) have beneficial effects on fertility, especially sperm quality, and they are therefore recommended as a potentially effective treatment for male infertility (Walczak-Jedrzejowska, Wolski, \& Slowikowska-Hilczer, 2013).

Several studies have reported that antioxidant therapy can reverse sperm DNA damage and improve other sperm parameters (Majzoub et al., 2017) In a meta-analysis, Omar et al. (2019) compared CoQ10 and L-carnitine with a placebo and concluded that antioxidants could protect against free radical damage in infertile men with higher ROS levels. Antioxidants were also found to improve spermatogenic function and sperm DNA integrity (Elumalai et al., 2009). Various clinical trials and systemic studies including the use of various combinations of antioxidants (L-carnitine, selenium, N-acetylcysteine, CoQ10, ubiquinol, vitamin $\mathrm{E}$, vitamin $\mathrm{C}$, and lycopene) in infertile men reported the beneficial effects of antioxidants on sperm concentration and motility and DNA integrity (Agarwal et al.,2019). Abad et al. (2013) also conducted a study to determine the effect of oral antioxidant therapy on the dynamics of sperm DNA fragmentation in a cohort of 20 infertile patients diagnosed with asthenoteratozoospermia. All subjects were given a combination of 1,500 mg L-carnitine, $60 \mathrm{mg}$ vitamin C, $20 \mathrm{mg}$ CoQ10, $10 \mathrm{mg}$ vitamin E, $10 \mathrm{mg}$ zinc, 200 microgram folic acid, 50 microgram selenium and 1 microgram vitamin B12 for a period of three months. The results showed that the rate of sperm with DNA damage was significantly reduced, and there was a significant increase in the concentration, motility, viability and morphology parameters of the semen analysis data. Furthermore, a significant improvement in DNA integrity was observed at all incubation points. The findings of that study were considered to indicate that antioxidant therapy helped preserve sperm quality not only in terms of important seminal parameters and basal DNA damage but also DNA integrity. In another study, Gopinath et al. (2013) noted that the administration of antioxidants in men with OAT resulted in a significant improvement in their sperm count and total motility at 90 days compared with the placebo.

Tremellen et al. (2007) conducted a prospective, randomized, double-blind, placebo-controlled study in 60 couples with severe male factor infertility. The participants were randomly assigned to take either one capsule of a combination containing $6 \mathrm{mg}$ lycopene, $400 \mathrm{IU}$ vitamin E, $100 \mathrm{mg}$ vitamin C, $25 \mathrm{mg}$ zinc, 26 microgram selenium, $5 \mathrm{mg}$ folate, and 1,000 mg of garlic or a placebo three months before their partners' in 
vitro fertilization or intracytoplasmic sperm injection (IVF or ICSI) cycle. The antioxidant group showed a statistically significant improvement in live pregnancy rates (38.5\%) compared to the control group (16\%). A meta-analysis by Lafuente et al. (2013) showed that treatment with CoQ10 resulted in a significant improvement in sperm motility and density, but no significant improvement was observed in live birth or pregnancy rates. In another study, combination therapy with carnitine, CoQ10, vitamin $\mathrm{E}$ and vitamin $\mathrm{C}$ for three to six months improved sperm concentration (Gvozdjáková et al., 2015).

In a systematic review published by Ross et al. in 2010, 17 randomized studies were selected to evaluate the effects of oral antioxidants on sperm quality and pregnancy rate in infertile men. The results showed that the treatment of infertile men with oral antioxidants could reduce seminal OS and improve sperm motility, but had a less predictable effect on sperm concentration and morphology. In addition, oral antioxidant therapy was associated with a significant improvement in spontaneous and assisted pregnancy rates in six of 10 randomized studies included in the analysis (Ross et al., 2010). In our study, the positive effects of two different combinations on sperm parameters were determined. Since there was no placebo group, it is not possible to comment on spontaneous pregnancy rates; however, there was a significant increase on the morphology values of both molecules.

Showell et al. (2011) evaluated whether supplementation with oral antioxidants would improve the results of assisted reproductive techniques when used in the male partners of couples undergoing assisted reproductive techniques (ART), and how antioxidants might affect the pregnancy rate, sperm parameters, and sperm DNA fragmentation. Data from 34 studies including a total of 2,876 couples were included in the analysis. Antioxidant treatment was associated with a statistically significant increase in the rates of live birth [odds ratio: $4.85,95 \%$ confidence interval $(\mathrm{CI}): 1.92-12.24 ; \mathrm{p}=0.0008]$ and pregnancy (OR: $4.18,95 \%$ CI: 2.65-6.59; $\mathrm{p}<0.00001$ ) compared to the control groups (Showell et al., 2011). In a recent review, Clark et al. (2013) examined 37 randomized controlled trials on complementary and alternative medicine, including antioxidants and nutritional supplements for the treatment of male infertility. Despite the presence of some preliminary evidence of the efficacy of antioxidant interventions among infertile patients, the authors emphasized the need for further research before the adoption of these modalities in routine clinical use (Clark et al. 2013). Similar conclusions were reached by Imamovic Kumalic et al. (2017), who reviewed 32 studies conducted from 2000 to 2013 and found that most confirmed the beneficial effect of antioxidants on at least one semen parameters, with the most prominent effect being on sperm motility, as well as the possible role of dietary supplements in the treatment of idiopathic OAT.

In a study by Kızılay et al (2019)., infertile patients who underwent varicocelectomy were given antioxidants together with surgical treatment, and it was determined that the antioxidant + surgical treatment group achieved statistically significant results in relation to semen parameters and spontaneous pregnancy. In another recent study, patients with high-grade varicoceles were randomized to surgical treatment and Lcarnitine supplementation groups, and favorable results were obtained in all sperm parameters with motility increasing from 21.7 to $35.4 \%$ and 33.9 to $47.5 \%$, respectively; normal sperm morphology from 46.3 to $60 \%$ and 56.6 to $69.7 \%$, respectively, and seminal volume from 3.5 to $4.2 \mathrm{ml}$ and 2.9 to $4.3 \mathrm{ml}$, respectively. The authors concluded that additional therapy was as effective as varicocelectomy in improving semen parameters, and could therefore be used as an alternative to surgery (Sofimajidpour, Ghaderi, \& Ganji, 2016). In a study by Busetto et al. (2018) the effect of treatment on varicocele-induced infertility was evaluated by applying an adjuvant antioxidant or placebo to 114 patients with and without varicoceles. A significant increase was found in semen parameters and pregnancy rates of the group that had received antioxidant supplementation for six months.

Many studies in the literature report positive results regarding different agents and application methods and different doses of antioxidant treatments. The groups given antioxidant treatments together with ART, after varicocelectomy, or in patients with varicocele were observed to have significant improvements compared to the untreated groups. Due to the methodological and clinical heterogeneity of these studies, it is difficult to make a comparison between the agents administered or to make a definitive conclusion on the optimal dose and duration for a given oral treatment. In our study, both antioxidant combinations, which differ in 
terms of content, providing significant results compared to the baseline values, shows that the use of this treatment in infertile patients is useful. However, the two molecules with different content screating similar effects raises further questions concerning the molecules contained in each combination and their dose.

The limitations of this study include the absence of a placebo group and an evaluation of sperm DNA damage not being undertaken.

\section{Conclusions}

Although it is not possible to make a definitive recommendation on antioxidant therapy for the treatment of idiopathic male infertility, increasing evidence from randomized trials and systematic reviews suggests that oral antioxidant supplementation can improve semen parameters and the probability of pregnancy. However, these studies are not sufficient and there is a wide variation in the treatment regimens used. Therefore, to select the optimal population that can benefit from oral antioxidant therapy and to evaluate the effect of standardized specific antioxidant doses on both spontaneous and assisted pregnancy rates, there is a need for larger randomized controlled studies to investigate different molecules and their combinations.

\section{Acknowledgment of funding}

There is no sources of funding for conducting the research.

\section{REFERENCES}

Abad C, Amengual M, Gosálvez J, Coward K, Hannaoui N, Benet J, et al. (2013) Effects of oral antioxidant treatment upon the dynamics of human sperm DNA fragmentation and subpopulations of sperm with highly degraded DNA. Andrologia, 45: 211-216.

Agarwal, A., Hamada, A., \& Esteves, S. C. (2012). Insight into oxidative stress in varicocele-associated male infertility: Part 1.Nature Reviews Urology , 9 , 678-690.https://doi.org/10.1038/nrurol.2012.197

Agarwal A, Saleh RA, Bedaiwy MA. (2003) Role of reactive oxygen species in the pathophysiology of human reproduction. Fertil Steril , 79:829-843.

Agarwal A, Parekh N, Panner Selvam MK, Henkel R, Shah R, et al. (2019) Male oxidative stress infertility (MOSI): proposed terminology and clinical practice guidelines for management of idiopathic male infertility. World J Mens Health, 37:296-312.

Agarwal A, Virk G, Ong C, du Plessis SS. (2014) Effect of oxidative stress on male reproduction. World $J$ Mens Health , 32:1-17.19:511-20.

Balercia, G., Regoli, F., Armeni, T., Koverech, A., Mantero, F., \& Boscaro, M. (2005). Placebocontrolled double blind randomized trial on the use of l-carnitine, l-acetylcarnitine or combined l-carnitine and l-acetylcarnitine in idiopathic asthenozoospermia. Fertility and Sterility , 84 (3), 662-671. https://doi.org/10.1016/j.fertnstert.2005.03.064

Busetto GM, Agarwal A, Virmani A, Antonini G, Ragonesi G, Del Giudice F, et al. (2018) Effect of metabolic and antioxidant supplementation on sperm parameters in oligo-astheno-teratozoospermia, with and without varicocele: A double-blind placebo-controlled study. Andrologia . 50(3). doi: 10.1111/and.12927.

Clark NA, Will M, Moravek MB, Fisseha S. (2013) A systematic review of the evidence for complementary and alternative medicine in infertility.Int J Gynaecol Obstet ., 122:202-6.

Cooper TG, Noonan E, von Eckardstein S, Auger J, Baker HW, Behre HM, et al. (2010) World Health Organization reference values for human semen characteristics. Hum Reprod Update , 16: 231-45.

Dorostghoal M, Kazeminejad SR, Shahbazian N, Pourmehdi M, Jabbari A. (2017) Oxidative stress status and sperm DNA fragmentation in fertile and infertile men. Andrologia, 49:e12762.

Durackova, Z. (2014). Free radicals and antioxidants for non-experts. In I. Laher (Ed.), Systems biology of free radicals and antioxidants(pp. 537-565). Berlin, Germany: Springer Verlag. 
Elumalai P, Krishnamoorthy G, Selvakumar K, Arunkumar R, Venkataraman P, Arunakaran JJR.(2009) Studies on the protective role of lycopene against polychlorinated biphenyls (Aroclor 1254)-induced changes in StAR protein and cytochrome P450 scc enzyme expression on Leydig cells of adult rats.Reprod Toxicol, $27: 41-5$.

Gopinath P, Kalra B, Saxena A, Malik S, Kochhar K, Kalra S, et al. (2013) Fixed Dose Combination Therapy of Antioxidants in Treatment of Idiopathic Oligoasthenozoospermia: Results of a Randomized, Double-blind, Placebo-controlled Clinical Trial. Int J Infertil Fetal Med , 4: 6-13.

Gvozdjáková A, Kucharská J, Dubravicky J, Mojto V, Singh RB. (2015) Coenzyme Q10, $\alpha$-Tocopherol, and Oxidative Stress Could Be Important Metabolic Biomarkers of Male Infertility. Dis mark, 2015: ID 827941.

Jungwirth A, Diemer T, Dohle GR, Giwercman A, Kopa Z, Krausz C, et al. (2013) members of the European Association of Urology (EAU) Guidelines Office. Guidelines on Male Infertility. In: EAU Gui de lines, edition presented at the 28th EAU Annual Congress, Milan.

Imamovic Kumalic S, PinterB. (2014) Review of clinical trails on effects of oral antioxidants on basicsemen and other parameters in idiopatic oligoasthenoteratoozospermia. Biomed Res Int ., 2014:426951. Doi: $10.1155 / 2014 / 426951$

Kizılay F, Altay B. (2019) Evaluation of the effects of antioxidant treatment on sperm parameters and pregnancy rates in infertile patients after varicocelectomy: a randomized controlled trial. Int $J$ Impot Res . ,31:424-431.

Lafuente R, González-Comadrán M, Solà I, López G, Brassesco M, et al. (2013) Coenzyme Q10 and male infertility: a meta-analysis. J Assis Reprod Gen , 30: 1147-1156.

Lenzi, A., Sgro, P., Salacone, P., Paoli, D., Gilio, B., Lombardo, F., et al. (2004). A placebo-controlled doubleblind randomized trial of the use of combined L-carnitine and L-acetyl- carnitine treatment in men with asthenozoospermia. Fertility and Sterility , 81(6), 1578-1584. https:// doi.org/10.1016/j.fertnstert.2003.10.034

Majzoub A, Agarwal A, Esteves SC. (2017) Antioxidants for elevated sperm DNA fragmentation: a mini review. Transl Androl Urol ., 6:649-53.

Nieschlag, E., Behre, H. M., \& Nieschlag, S. (2010). Male reproductive health

and dysfunction. Andrology. Berlin, Germany: Springer Verlag.

Omar MI, Pal RP, Kelly BD, Bruins HM, Yuan Y, Diemer T, et al. Benefits of Empiric Nutritional and Medical Therapy for Semen Parameters and Pregnancy and Live Birth Rates in Couples with Idiopathic Infertility: A Systematic Review and Meta-analysis. Eur Urol 2019;75:615-25.

Ross C, Morriss A, Khairy M, Khalaf Y, Braude P, Coomarasamy A, et al. (2010) A systematic review of the effect of oral antioxidants on male infertility. Reprod Biomed Online. , 20(6):711-23. doi: 10.1016/j.rbmo.2010.03.008.

Showell MG, Brown J, Yazdani A, Stankiewicz MT, Hart RJ. (2011) Antioxidants for male subfertility. Cochrane Database Syst Rev. 2011 Jan 19;(1):CD007411. doi: 10.1002/14651858.CD007411.pub2. Update in: Cochrane Database Syst Rev. 2014;12:CD007411.

Sharlip ID, Jarow JP, Belker AM, Lipshultz LI, Sigman M, Thomas AJ, et al. (2002). Best practice policies for male infertility. Fertil Steril 77(5):873-82. doi: 10.1016/s0015-0282(02)03105-9.

Sofimajidpour, H., Ghaderi, E., \& Ganji, O. (2016). Comparison of the effects of varicocelectomy and oral L-carnitine on sperm parameters in infertile men with varicocele. Journal of Clinical and Diagnostic Research , 10(4), PC07-PC10.

Tremellen K, Miari G, Froiland D, Thompson J. (2007) A randomised control trial examining the effect of an antioxidant (Menevit) on pregnancy outcome during IVF-ICSI treatment. Aust $N$ Z J Obstet Gynecol, 
47: 216-221.

Walczak-Jedrzejowska, R., Wolski, J. K., \& Slowikowska-Hilczer, J. (2013). The role of oxidative stress and antioxidants in male fertility. Central European Journal of Urology , 66(1), 60-67. https://doi.org/10.5173/ ceju.2013.01

World Health Organization. WHO Manual for the Standardized Investigation and Diagnosis of the Infertile Couple. Cambridge: Cambridge University Press, 2000. 